\title{
The art of producing quality evidence-based systematic reviews
}

\section{Felix S F Ram}

$\mathrm{T}$ he volume of data that need to be considered by health care practitioners and researchers is expanding at an exponential rate, and as a result of this, health professionals are no longer able to keep up with all publications in their area of interest. In even a single area, it is not unusual for the number of published trials to run into dozens or even hundreds. This has created difficulties in ensuring that clinical practice is based on reliable sources of information and research. In addition to this large volume, clinicians often have to deal with unclear, confusing and contradictory research results. Looked at individually, each trial may offer little insight into effectiveness; but when taken together, a clearer picture will emerge. This situation has created the need for summarising research results.

Traditionally we have relied on narrative reviews of health care interventions to summarise the research in a "digestible" form. Narrative reviews have always been part of the medical literature. Respected leaders, experts in their field, have sought to collate existing knowledge and publicise these summaries.

Unfortunately, such attempts at synthesis have not always been as rigorous as might have been hoped. One obvious problem is that, traditionally, reviewers rarely began with an open mind as to the likely recommendations. Indeed, those involved in developing a narrative review may well have started a review precisely because of their accumulated experience and professional opinions. If strong prior beliefs are held then a dispassionate review of evidence will be difficult to achieve. Even if the reviewer does begin with an open mind, traditional narrative reviews are rarely explicit about how studies are selected, assessed and integrated. Thus, the reader is often not able to replicate the process and is also generally unable to assess the likelihood of prior beliefs or other biases, which usually clouds the narrative review process. For all this, narrative reviews still remain widespread and influential. The lack of rigour in the creation of reviews went largely unremarked until the late 1980s when several commentators exposed the inadequacies of the process and the consequent bias in recommendations. ${ }^{1,2}$ Not least of the problems was that small but important effects were being missed, different reviewers were reaching different conclusions from the same research base. ${ }^{3}$ The inadequacy of traditional reviews and the need for a rigorous systematic approach were emphasised in 1992 with the publication of two landmark papers reporting devasting findings. ${ }^{4,5}$ The first paper reported that if original studies of the effects of clot busters after heart attacks had been systematically reviewed the benefits of therapy would have been apparent as early as the mid-1970s. The second paper showed that textbooks and narrative reviews were highly inadequate in summarising the current state of knowledge. In addition, an analysis of manuscripts in five general medical journals showed that the results of new studies are only very rarely presented in the context of reviews of relevant earlier studies. ${ }^{6}$ Recognising this shortcoming in publications, the British Medical Journal began publishing the "What is already known on the topic" and "What this article adds" boxes with all its articles. This is an attempt certainly in the right direction by the British Medical Journal to acknowledge the cumulative nature of scientific evidence and to review research with the results of new studies set in the context of other previously known relevant research.

All three papers mentioned above ${ }^{4-6}$ showed that there was much knowledge to be gained from collating existing research, but that traditional approaches had largely failed to abstract this knowledge. What was needed was the same rigour in secondary research as was expected from primary research. Systematic reviews have since emerged to fulfill this need by providing comprehensive and unbiased summaries of the research. These systematic reviews bring together large numbers of individual studies in a single document and some organisations involved in such research, like the Cochrane Collaboration, also regularly update their reviews with continually emerging evidence.

Systematic reviews are designed to help the clinician base their clinical decision on the best available evidence. Systematic reviews include a comprehensive search strategy and a predetermined and explicit method to appraise and synthesize the information from individual studies. Systematic reviews are rapidly superseding narrative reviews in almost all areas of health care. However, the quality of a review, and so it's worth, depends on the extent to which scientific review methods are used to minimise the risk of error and bias. It is the use of these explicit and rigorous methods that distinguish systematic reviews from the traditional narrative reviews. Highquality systematic reviews take great care to find all relevant studies published and unpublished, assess each study, synthesise the findings from individual studies in an unbiased way and present a balanced and impartial summary of the findings with due consideration of any flaws in the evidence.

The steps considered most important in conducting high-quality quality systematic reviews are summarised in Table 1. Examples of many highquality reviews are available both in journals (e.g. Evidence Based Medicine, British Medical Journals) and from electronic sources such as the Cochrane Library (http://www.cochrane.org), which has recently been made freely available to all in the UK.

Systematic reviews now appear at the top of the

\author{
Dr Felix S F Ram \\ Research Fellow in \\ Respiratory Medicine \\ Correspondence to: \\ Dr Felix S F Ram \\ Cochrane Airways Group \\ St. Georges Hospital \\ Medical School \\ Department of \\ Physiological Medicine \\ Level 0, Jenner Wing \\ Cranmer Terrace, Tooting \\ London, SW17 0RE
}

Email: fram@sghms.ac.uk

Date submitted: 28/10/02 Date Accepted: 08/11/02

Prim Care Resp J 2002: 11(4);117-119 
Table 1: Steps in conducting a high-quality systematic review (adapted from Egger et al) ${ }^{8}$.

\section{Formulate review question}

- Clearly define the clinical question being addressed

\section{Define inclusion and exclusion criteria}

- participants

- interventions and comparisons

- outcomes

- study designs and methodological quality

\section{Locate studies}

Search strategy should consider the

following:

- Cochrane Controlled Trials Register (CENTRAL)

- electronic databases of individual journals and ongoing trials register

- checking of reference list in papers

- handsearching of key journals

- personal communication with experts in field

\section{Select studies}

- have eligibility checked by more than one reviewer

- develop strategy to resolve disagreements

- keep log of excluded studies, with reasons for exclusion

\section{Assess study quality}

- consider assessment by more than one reviewer

- use simple checklists rather than quality scales
- always assess concealment of treatment allocation, blinding and handling of patient attrition

- consider blinding of reviewers to authors, institutions and journals

\section{Abstract data}

- design and pilot data abstraction forms

- consider data abstraction by more than one reviewer

- consider blinding of reviewers to authors, institutions and journals

\section{Analyse and present results}

- tabulate results from individual studies

- examine forest plots

- explore possible sources of heterogeneity

- consider meta-analysis of all trials or subgroups of trials

- perform sensitivity analyses, examine funnel plots

\section{Interpret results}

- consider limitations, including publication and related bias

- consider strength of evidence

- consider applicability

- consider numbers-needed-to-treat to benefit / harm

- consider economic implication

- consider implications for future research

'hierarchy of evidence' (Table 2). This reflects the fact that when well conducted they should give us the best possible answer. However, not all published systematic reviews are of high quality and like any piece of research, a systematic review may also be poorly conducted. There are many tools freely available for critically appraising and conducting systematic reviews (see Appendix 1) and there are also regular courses conducted by the UK Cochrane Centre (Oxford) and the NHS Centre for Reviews and Dissemination (University of York). An incredibly helpful and user-friendly book compiled by the British Medical Journal

(http://www.systematicreviews.com) and considered by most as the "bible for systematic reviewing" would be a good starting point for any person considering undertaking a systematic review. $^{7}$

A word of caution, undertaking a systematic review in no easy task and in order to conduct a quality systematic review, requires good understanding of the principles behind producing such reviews. The strength of the evidence provided by an individual review is dependent on the ability of the review design to minimise the possibility of bias and to maximise attribution. However,

Table 2: A summarised version of the accepted hierarchy of evidence in descending order of strength.

Systematic reviews and meta analyses of randomised controlled trials

$\downarrow$ Randomised controlled trials

$\downarrow$ Observational studies

$\downarrow$ Non-experimental studies

$\downarrow$ Expert opinion.

$\downarrow$ Clinical experience

\section{Table 3: Some key elements when critically appraising or conducting a} systematic review.

1. Did the review address a focused and well-defined clinical question?

2. Were the criteria for study selection clearly described, appropriate and fairly applied?

3. Is it unlikely that important, relevant studies were missed?

4. Is there inappropriate aggregation of studies (both clinically and statistically)?

5. Was study quality assessed by independent reviewers?

6. Are assessments of studies reproducible?

7. Was missing information sought from the original study investigators?

8. Were the results similar from study to study?

9. Were all clinically important outcomes considered?

10. Were the overall findings assessed for their robustness?

11. Was the play of chance assessed?

12. Are the recommendations based firmly on the quality of evidence presented?

13. Are the conclusion supported by the evidence presented? the strength of evidence provided by the review is also influenced by how well it was designed and carried out. Failure to give due attention to key aspects of review methodology increases the risk of bias or confounding and thus reduces its reliability and credibility. Some key methodological issues that require to be addressed when assessing or conducting a systematic review are summarised in Table 3 .

Subscribers to the Primary Care Respiratory Journal and members of the General Practice Airways Group who may be interested in conducting a systematic review on a particular topic in respiratory medicine are free to contact the author for further advice and guidance. 
For personal use only.

Not to be reproduced without the permission of the Primary Care Respiratory Journal

\section{Appendix 1:}

Some useful links for information regarding the conduct of quality evidence-based systematic reviews.

\section{Cochrane Library training materials}

This site offers a set of Word documents and a PowerPoint presentation designed for training users in the efficient use of The Cochrane Library. These materials are regularly updated for each issue of The Cochrane Library.

http://www.york.ac.uk/inst/crd/cochlib.htm

\section{Cochrane Library Users Group}

Opportunity to send feedback about The Cochrane Library; results of surveys of users of The Cochrane Library; information about the group http://www.york.ac.uk/inst/crd/clug.htm

\section{Centre for Evidence-Based Medicine} Oxford, UK

http://www.minervation.com/cebm//

\section{Health Information Research Unit}

Evidence-Based Health Informatics

McMaster University, Hamilton, Canada http://hiru.hirunet.mcmaster.ca/

\section{National Health Service (UK) - Health Technology Assessment}

The HTA programme actively seeks out the questions that meet the NHS's research needs, commissions research to meet those needs, and communicates the findings to the NHS and its users. http://www.hta.nhsweb.nhs.uk

\section{QUOROM Statement: Quality of Reporting of} Meta-analysis

A checklist and flow diagram for improving the quality of reports of meta-analysis of randomised controlled trials.

http://www.consort-statement.org/QUOROM.pdf

\section{References}

1. Mulrow CD. The medical review article: state of the science. Ann Intern Med 1987; 106:485-8.

2. Teagarden JR. Meta-analysis: whither narrative review? Pharmacotherapy 1989; 9:274-84.

3. Spector TD, Thompson SG. The potential and limitations of meta-analysis. J Epidemiol Community Health 1991;45:89-92.

4. Lau J, Antman EM, Jimenez-Silva J, Kupelnick B, Mosteller F, Chalmers TC. Cumulative meta-analysis of therapeutic trials for myocardial infarction. $N \mathrm{Engl}$ $J$ Med 1992; 327:248-54.

5. Antman EM, Lau J, Kupelnick B, Chalmers TC. A comparison of results of meta-analyses of randomized control trials and recommendations of clinical experts. JAMA 1992;268:240-8.

6. Clarke M, Chalmers I. Discussion sections in reports of controlled trials published in general medical journals: islands in search of continents? JAMA 1998;280:280-2.

7. Egger M, Smith GD, Altman DG. Systematic reviews in health care. Meta-analysis in context. 2nd ed. London: BMJ Publishing Group, BMA House, Tavistock Square, WC1H 9JR, 2001.

8. Egger M, Smith GD. Principals of and procedures for systematic reviews. In: Egger M, Smith GD, Altman DG, editors. Systematic reviews in health care. Meta-analysis in context. 2nd ed. London: BMJ Publishing Group, BMA House, Tavistock Square, WC1H 9JR, 2001. 